\title{
ENCUENTROS Y DESPEDIDAS CON LESTER EMBREE
}

\section{ENCOUNTERS AND FAREWELLS WITH LESTER EMBREE}

Carlos Belvedere

Universidad de Buenos Aires

cbelvede@ungs.edu.ar

E assim chegar e partir...

São só dois lados Da mesma viagem $O$ trem que chega É o mesmo trem Da partida...

A hora do encontro É também, despedida.

(Milton Nascimento, Encontros e Despedidas)

Conocí a Lester Embree en 1999. Roberto Walton lo había invitado al Encuentro Nacional de Fenomenología y Hermenéutica que organiza todos los años en la Academia Nacional de Ciencias de Buenos Aires. Lejos estaba entonces de comprender la gravitación que ese acontecimiento tendría en mi vida.

Todavía recuerdo su imponente figura apoltronada en la antesala de la Academia. Una sonrisa afable encaramada a su fornida silueta me tendía una invitación al diálogo. Aunque recién entonces nos conocíamos personalmente, de algún modo yo ya lo "conocía" porque sus libros y artículos me habían influido profundamente. Mi tesis de doctorado había estado dedicada a Schutz, y dos de 
los principales pilares sobre los que se sostenía eran Worldly Phenomenology y Schutzian Social Science ${ }^{2}$, ambos libros compilados por Lester. También había trabajado minuciosamente el cuarto volumen de la obra de Schutz editado por él, junto con Helmut Wagner, George Psathas y Fred Kersten. Si algo podía ambicionar por aquel entonces era participar como autor en alguna de sus compilaciones. En eso consistía mi hybris. Por megalómanas que fueran mis pretensiones de "argentino", jamás pensé que tendría la fortuna de conocerlo personalmente y de recibir sus sabios consejos durante casi veinte años. Aquel primer encuentro me marcó, tanto en lo académico como en lo personal.

Lester era una persona de virtudes contrastantes. No hablo de un balance de virtudes y defectos sino de una feliz combinación de cualidades reunidas de modo inesperado. La fuerza de sus convicciones, que su voz potente expresaba con elocuencia, lejos de intimidar y demarcar posiciones excluyentes, propiciaba el encuentro entre los interlocutores menos pensados. Una disposición amigable y ecuménica se expresaba en sus alocuciones tajantes, que solían combinar la ironía con la confianza ciega en el diálogo, pues consideraba que la fenomenología sólo es posible en el encuentro de quienes la practicamos en sus más diversas formas. Además, tenía un ojo clínico infalible. A pocos días habernos conocido ya me había dado uno de sus grandes consejos, que todavía atesoro.

Walton tuvo la gentileza de avisarme de la visita de Lester con anticipación y de sugerirme que lo invitara a presentar una charla en la Facultad de Ciencias Sociales de la Universidad de Buenos Aires. No pude desaprovechar semejante oportunidad. El 24 de septiembre, en el Instituto de Investigaciones Gino Germani (perteneciente a la Facultad), Lester presentaba un tema en el que - veremos luego- seguiría interesado a lo largo de su vida, especialmente en sus últimos escritos. Ante una selecta pero nutrida concurrencia, abordó la cuestión de "Los grupos en Schutz: La estructura significativa concreta del mundo socio-histórico". Luego de la charla fuimos a comer. Como anfitrión, pedí la cuenta y me dispuse a pagar. Lester se opuso amablemente y me dio allí la primera lección de vida. Era, por cierto, un mandato, pero uno sabio y generoso.

\footnotetext{
${ }^{1}$ Lester Embree (ed.), Worldly Phenomenology: The Continuing Influence of Alfred Schutz On North American Human Science, Washington D.C.: Center for Advanced Research in Phenomenology and University Press of America, 1988.

2 Lester Embree (ed.), Schutzian Social Science, Dordrecht: Kluwer Academic Publishers, 1999. 
Tras agradecer la invitación y elogiar el establecimiento (que, de todos modos, no habrá sido de los más selectos que visitó en su vida de viajero incansable), insistió en pagar y me dijo que, como retribución, debía invitar a uno de mis estudiantes a comer a algún lugar como ese. Fue un gesto que me impresionó. Muchos años después, en uno de los innumerables congresos en los que coincidimos, le recordé la anécdota. Aunque la había olvidado, la idea le seguía pareciendo correcta y la obligación contraída, vigente. Me preguntó si había cumplido. Tuve la dicha de responder que sí, y que lo había hecho en reiteradas ocasiones.

La charla que había dado ese día también me impactó de un modo duradero. Abordó una de las cuestiones más polémicas en ciencias sociales. Se refirió a las entidades colectivas. No se apegó a la idea dominante de que Schutz negaba su existencia sino que - para mi sorpresa - argumentó que el grupo preexiste al individuo.

No sonaba muy schuziana la idea, aunque sí lo era. Con fidelidad, aunque también con libertad y originalidad, Lester mostró que es posible encontrar en la obra de Schutz una auténtica sociología de los grupos que difiere en aspectos sustantivos del individualismo metodológico con el cual, de un modo habitual y dogmático, se lo suele relacionar.

No era una simple provocación. Lester se mostraba convencido de que el individuo es derivado a partir del grupo. Y sostuvo esta convicción a lo largo de los años, tal como pude constatar al seguir el desarrollo de su obra.

Hasta sus últimos escritos, Lester continuó trabajando esta idea ${ }^{3}$, profundizando y sofisticando los argumentos presentados en aquella conferencia. De esta reelaboración surgieron dos argumentos principales: uno, metodológico, según el cual Schutz no fue el individualista metodológico que muchos creen; otro, ontológico, según el cual los individuos son abstracta abstraídos a partir de los grupos, que son concreta 4 .

\footnotetext{
${ }^{3}$ Esta continuidad puede apreciarse en la siguiente secuencia; Lester Embree, "Los grupos en Schutz: La estructura significativa concreta del mundo socio-histórico", conferencia dictada en el Instituto de Investigaciones Gino Germani de la Universidad de Buenos Aires, el 24 de septiembre de 1999, traducción de Claudia Martínez U., mimeo; Lester Embree, "From 'We' to 'I' and Back: Still Learning from the New School", The 41st Annual Meeting of The Husserl Circle, New York City: The New School for Social Research, 2010, pp. 37-46; Lester Embree, "Groups in Schutz: The Concrete Meaning Structure of the SocioHistorical World", PhaenEx, vol. 6, 1 (2011) 1-11; Lester Embree, The Schutzian Theory of the Cultural Sciences, Cham/Heidelberg/New York/Dordrecht/London: Springer, 2015.

${ }^{4}$ Lester Embree, "Groups in Schutz...", op. cit., pp. 1-11.
} 
Lo que me impresionó de Lester es que, sin abandonar la fenomenología, podía hablar de entidades colectivas - lo que para otros sería un tema tabú. En un sentido muy schutziano, aunque diciendo algo que Schutz jamás dijo de ese modo, sostenía que hay vida colectiva y que, para alcanzarla, el individualismo metodológico resulta insuficiente.

Como dije, la posición metodológica de Lester contradecía el consenso predominante de que Schutz había sido un individualista metodológico. Lester argumentaba que esta concepción fue prontamente abandonada por Schutz. Por cierto, el individualismo metodológico puede encontrarse en su opera prima, donde elogia a Georg Simmel y a Max Weber por haberlo introducido en las ciencias sociales. Incluso parece haberlo puesta en práctica al oponerse al supuesto "colectivismo metodológico" de Talcott Parsons, por ejemplo5. Pero esta quirúrgica delimitación no hace más que resaltar el hecho de que a Schutz el individualismo metodológico no le duró mucho...

Hay un giro en la obra de Schutz que, quizá por motivos ideológicos o a lo mejor por mera desatención, ha pasado inadvertido para todos menos para Lester. Schutz abandona el individualismo metodológico a poco de haberlo adoptado; abandono que ocurre ni más ni menos que en el transcurso de su primer libro, que comienza con esta perspectiva y culmina con la contraria. A partir de entonces, se fue volviendo cada vez más consciente de que siempre partimos de un mundo intersubjetivo y por lo tanto nunca estamos solos. La sociedad no es algo que pueda reconstruirse a partir de interacciones entre individuos porque el individuo es una abstracción de la única realidad, que es la vida concreta de los grupos.

En consecuencia, ni el individualismo metodológico ni la egología por sí misma pueden acceder a ella. Antes de operar la reducción egológica - sostiene Lester- nos encontramos en actitud intersubjetiva, lo cual significa que somos subjetividades que se encuentran unas junto a otras y comparten un mundo objetivo común o, mejor dicho, un mundo "público". En otras palabras, ante todo somos miembros de un "Nosotros"6.

Así que, aunque muchos creen que Schutz pensaba que lo concreto es el individuo y que lo colectivo es un ensamble de individuos, más bien ocurrió lo

\footnotetext{
${ }^{5}$ Lester Embree, "From 'We' to 'I' and Back...", op. cit., p. 40.

${ }^{6}$ Ibid.
} 
contrario: que, para él, el individuo era un abstractum obtenido a partir de la "vida colectiva concreta"7. Toda concepción del mundo social como estructura de individuos - nos dice Lester- es producto de una abstracción y, por lo tanto, resulta abstracta.

En efecto, el argumento de Lester - con el cual coincido plenamente- es que para Schutz la idea de que los individuos existen por sí mismos, separados unos de otros, es una "abstracción ficticia" porque, en realidad, lo concreto son los grupos. Los individuos, en cambio, si se los concibe al margen de sus membresías grupales, son meras abstracciones ${ }^{8}$ ya que siempre somos miembros de algún grupo; y esto es algo que no queda claro cuando se concibe el mundo social como una estructura de individuos ${ }^{9}$.

Si el mundo social no puede concebirse de este modo, es porque contiene grupos relacionados entre sí de diversas maneras y que son, precisamente, lo fundamental en tanto son concretos y no abstractos, como los individuos. Luego, la estructura fundamental del mundo social es la de los grupos, de los cuales se puede decir (al menos "metafóricamente") que viven, nacen y mueren ${ }^{10}$. Hay, entonces, una vida colectiva concreta, la cual habría sido advertida no solo por Lester sino también por Schutz, cuya ontología social "incluye colectividades sociales"11.

Esta es, principalmente, la conclusión que extrae Lester, a sabiendas de que está yendo más allá de la letra de Schutz, aunque también con la convicción de no traicionar su espíritu. Los grupos son el sujeto colectivo de la vida social. Funcionan como el yo [self] en la estructura del individuo y pueden relacionarse con otros de un modo análogo a como el individuo se relaciona con sus socios, predecesores, contemporáneos y sucesores ${ }^{12}$. Más aún, los miembros de esos grupos pueden tener y compartir sentidos subjetivos y objetivos comunes y así

\footnotetext{
7 Lester Embree, The Schutzian Theory of the Cultural Sciences, pp. 125s. Véase también: "Groups in Schutz...", Op. cit., p. 5; y "Los grupos en Schutz...", op. cit., p. 3.

8 Lester Embree, The Schutzian Theory..., op. cit., p. 43. Véase también: "Groups in Schutz...", op. cit., p. 6; "From 'We' to 'I' and Back: Still Learning from the New School", p. 44; y "Los grupos en Schutz...", op. cit. La estructura significativa concreta del mundo socio-histórico", pp. 3s.

9 Lester Embree, The Schutzian Theory..., op. cit., p. 125 (el subrayado es de Embree). Véase también: "Groups in Schutz..." op. cit., p. 5.

${ }^{10}$ Lester Embree, The Schutzian Theory..., op. cit., p. 129. Véase también: "Groups in Schutz.." op. cit., p. 11; y "Los grupos en Schutz..." op. cit., pp. 5s.

${ }^{11}$ Embree, Lester, The Schutzian Theory... op. cit., p. 124. Véase también: "Los grupos en Schutz..." op. cit., p. 2; y "Groups in Schutz...", op. cit, p. 3.

12 Lester Embree, The Schutzian Theory..., op. cit., p. 124. Véase también: "Groups in Schutz..." op. cit., p. 3; y "Los grupos en Schutz..." op. cit., p. 2.
} 
constituir una auténtica "relación-Nosotros", en el sentido que le da Schutz a esta expresión ${ }^{13}$.

El ejemplo más conveniente al respecto es el de los grupos primarios, que son - según observa Lester- "colectividades consociales"14 y nos permiten apreciar más claramente la analogía entre una estructura social de individuos y otra de grupos. En el encuentro cara a cara, un grupo de consoncios puede ser análogo al yo en la estructura social de los individuos. En ese caso, este nosotros sería un sujeto. En cuanto tal, un grupo primario tendría un punto de vista colectivo y podría tener sentidos e interpretaciones compartidas, incluidas las autointerpretaciones, a partir de las cuales puede ejercer influencias internas y externas ${ }^{15}$.

A fin de comprender mejor esto, Lester acuña la expresión "grupos Vosotros", en analogía con la relación-Tú descripta por Schutz. Los grupos primarios serían de esta clase pues podrían tener "vidas internas colectivas de comprensión mutua e interacción", así como "una situación común "que definen e interpretan en base a significados subjetivos compartidos ${ }^{16}$. Además, estos grupos pueden entrar en relación con otros "grupos nosotros" y con "grupos ellos" o "vosotros". Todo lo cual implica que los grupos tienen vida interior colectiva ${ }^{17}$.

Como ya mencioné, nada de esto puede captar el individualismo metodológico. Lester lo sabe, y por eso adopta una estrategia distinta. No compartía la idea de muchos respecto de que el carácter meramente metodológico del individualismo de Weber y, presuntamente, de Schutz, no implica negar la existencia de entidades colectivas pues simplemente indica un modo de abordaje, no una concepción sustantiva de lo social. ¿Cómo podría nuestra metodología contrariar nuestra ontología? No resulta convincente una creencia en entidades colectivas que predique como única vía de acceso a ellas el recurso al individuo. Además, contradice la idea - que hemos tomado de Lester- de que existe una experiencia subjetiva propia de los sujetos colectivos. Será a esa experiencia, y no simplemente un agregado de experiencias individuales, a lo que habrá que acceder.

\footnotetext{
13 Lester Embree, The Schutzian Theory..., p. 125. Véase también: "Groups in Schutz...", op. cit., p. 4; y "Los grupos en Schutz..." op. cit.", pp. 2s.

14 Lester Embree, The Schutzian Theory..., op. cit., p. 127.

15 Ibid. Véase también: "Groups in Schutz...", p. 7.

${ }^{16}$ Lester Embree, The Schutzian Theory..., op. cit., p. 127. Véase también: "Groups in Schutz...", op. cit., p. 7.

17 Lester Embree, The Schutzian Theory..., p. 127. Véase también: "Groups in Schutz...", op. cit.,7. 
Para ello, será preciso adoptar la perspectiva que Lester insistentemente (no sólo en sus escritos sino también en incontables conferencias y debates) definió como "primera persona del plural"18.

Esta convicción de larga data encuentra una de sus expresiones magistrales en la presentación de Lester durante el $41^{\mathrm{er}}$ encuentro anual del Husserl Circle, donde se pregunta si la tan mentada "perspectiva en primera persona" es algo más que una terminología tomada de la lingüística y si no habría que definirla como "plural" en vez de singular ${ }^{19}$.

Lester consideraba que la perspectiva en primera persona del singular era consecuencia de un "tonto individualismo puesto de rodillas" característico del eurocentrismo y contrapuesto a la tendencia dominante en las culturas asiáticas, que siempre consideran a las personas como miembros de los grupos a los que pertenecen ${ }^{20}$. Para Lester, entonces, es preciso - siguiendo el mandato de Schutz- partir de la intersubjetividad; con lo cual la perspectiva en primera persona del plural se vuelve imperiosa ${ }^{21}$.

A pesar de que Lester remitía estas ideas a Schutz, bien sabemos que le son propias y que significan una originalísima contribución a la sociología fenomenológica. Sabía que estaba nadando contra la corriente al romper la dogmática asociación entre sociología fenomenológica e individualismo metodológico. Si esta metodología deja escapar la experiencia que de sí tienen los grupos, tanto como el sentido (subjetivo y objetivo) de sus acciones e interacciones, entonces estamos ante la necesidad de un nuevo abordaje que no oculte que el mundo social es un mundo de grupos y que los individuos nunca existen por sí mismos sino siempre como miembros de algún grupo.

Nótese que Lester encontró un modo fenomenológico de referirse a sujetos colectivos sin incurrir en mistificaciones metafísicas. Los grupos no son entidades imaginarias sino sujetos colectivos concretos, integrados por individuos o por otros grupos que pueden compartir sentidos subjetivos y objetivos y actuar unos sobre otros e influirse mutuamente. $Y$ a una experiencia colectiva no puede accederse sin un abordaje que también sea colectivo. Por eso la perspectiva que

\footnotetext{
18 Ibid.

19 Embree, Lester, "From 'We' to 'I' and Back...", op. cit., 40.

20 Ibid., p. 41.

21 Ibid., p. 40.
} 
ha de asumir el sociólogo fenomenológico no es la perspectiva en primera persona del singular sino del plural.

Claro que esto es más fácil decirlo que hacerlo; pero la dificultad de la empresa no le quita su valor. Ni la "erudición" ni la "filología" que Lester tan ácidamente cuestionó toda su vida bastarán para ello. Habrá que encontrar otro camino a las cosas mismas que nos dé acceso a los "hechos sociales" - esas cosas tan peculiares que la sociología, a veces infructuosamente, viene persiguiendo desde su fundación. Por fortuna y por desgracia, en esto Lester nos ha dejado mucho que pensar...

Me reencontré con Lester muchas veces. Uno de los efectos que tuvo su influencia en mí fue incentivarme a participar regularmente de congresos internacionales, empezando por el segundo encuentro de la Organización de Organizaciones Fenomenológicas (OPO) - una de las tantas instituciones que Lester contribuyó a fundar-, celebrado en la ciudad de Lima en el año 2005. Literalmente, aquel encuentro me abrió al mundo de la fenomenología.

Entre los numerosos colegas que allí conocí se encontraba Chung-Chi Yu. Lester lo presentó como: our guy in Taiwan. Ocasionalmente, Lester utilizaba un lenguaje al estilo de James Bond... Era la versión lúdica de su geopolítica fenomenológica. Más allá del sentido de aventura que la frase introducía, ponía de manifiesto su arraigada convicción de que lo más valioso que un congreso puede ofrecer es el encuentro entre colegas (o, si se prefiere, la "conexión", para continuar la alegoría...). Tan es así que, como bien se sabe, Lester valoraba más la charla en los pasillos y en las recepciones que el intercambio formal en ocasión de la lectura de ponencias. De ahí que las reuniones que organizaba o patrocinaba solían incluir una cena de camaradería, además de oportunas pausas para el café.

Volví a ver a Lester en el IX Congreso Internacional de Fenomenología y II Jornadas Ibéricas de Fenomenología en Segovia, en 2009. Luego, en el cuarto encuentro de OPO, realizado también en Segovia durante 2011. Coincidimos en dos encuentros (2011 y 2013) de The Interdisciplinary Coalition of North American Phenomenologists (ICNAP) y, durante 2009, en la conferencia international Phenomenology, Social Sciences, and the Arts, en la Universidad de Constanza. Pero donde más asiduamente lo traté fue en las reuniones anuales de la Society for Phenomenology and the Human Sciences (SPHS).

Desde 2011 hasta 2015, cada año nos reencontramos allí, donde Lester era una de las figuras históricas de mayor preponderancia. Su voz siempre era oída 
y su opinión, respetada. También eran festejadas sus humoradas, como aquella vez en que Jochen Dreher presentaba el libro de Erik Garrett, Why do we go to the Zoo? Dreher comenzó a enumerar los zoológicos que había visitado con sus hijos, a los cuales, desde el fondo de la sala, Lester agregó una asociación amiga de fenomenólogos que circunstancialmente se reunía en el mismo edificio que nosotros.

En los pasillos y sesiones de SPHS intercambiamos mucho más que chistes de ocasión. Lester tenía su propia comprensión de la fenomenología, que entregaba en pequeñas dosis, como grageas. Es bien conocida su preferencia por la fenomenología mundana [worldly phenomenology]. Quizá sea menos difundido que ella implica mucho más que un apego a la actitud natural de inspiración schutziana. En uno de esos corredores me dijo que la reducción fenomenológica está sobrevalorada [oversold]: I refrain myself from judging. So what? Y tras una de las sesiones en las que me tocó exponer, dijo que seguía a Aron Gurwitsch en la idea de que lo transcendental no es más que una función de la conciencia. Además, impulsaba entusiastamente la idea de Dorion Cairns de que lo que Husserl llama pasividad se comprende mejor como automaticidad: as a synthesis that runs on its own. A esta definición, Lester le agregaba otra, de su propia cosecha. En vez de "actos", prefería hablar de "operaciones" pues ello volvía -según su parecer- más tajante y sencilla la distinción entre actividad y pasividad 22 .

Otra de las organizaciones que Lester sostuvo desde sus inicios, The International Alfred Schutz Circle for Phenomenology and Interpretive Social Science, fue escenario de reiterados encuentros. Resultó memorable la reunión fundacional, que Lester tan delicadamente organizó en la New School for Social Research de Nueva York, en 2012; precisamente, en la institución donde Schutz impartiera sus clases. Luego volvimos a tenerlo en Buenos Aires gracias a una ayuda para viajes de la Fundación Williams, que también nos dio sede para el segundo encuentro del Círculo, en 2014.

Lester tuvo a su cargo una conferencia magistral en la que se refirió a "La verificación en Schutz". Como era uno de los organizadores del congreso, tuve ocasión de retribuirle la gentileza a Roberto Walton invitándolo a dar otra de las

22 Lester Embree, "Some Reflective Analysis of Recollecting", Bulletin d'analyse phénoménologique, vol. VII, 4 (2011), p. 6. http://popups.ulg.ac.be/bap.htm 
conferencias magistrales, que versó sobre "La historicidad en Edmund Husserl y Alfred Schutz". Por supuesto, Lester estaba allí. El debate que siguió fue uno de los más cautivantes que he presenciado. Tomó la palabra y comenzó expresándole al conferencista lo complacido que estaba de descubrir "cuánto sabes de Schutz". Lo que siguió fue, a pesar de los refranes sapienciales de Lester, un derroche de erudición. Volvimos a vernos para el tercer encuentro, en 2016; ocasión que también sería memorable, aunque por otros motivos.

Por gracia, o ironía, del destino, el último congreso al que asistimos nos encontró debatiendo el mismo tema que en nuestro primer encuentro; solo que con una desfavorable inversión: quien disertaba sobre los grupos en Schutz en esta ocasión era yo.

Comencé diciendo que mi ponencia se basaba extensamente en los trabajos de Lester, quien estaba allí, en la mesa de los moderadores de la sesión, sonriéndome con aprobación mientras yo explicaba que mi objetivo era definir la sociología fenomenológica como una ciencia que se ocupa de la concepción relativa natural del mundo que tienen los grupos humanos, continuando la dirección que Schutz le imprimiera a esta disciplina a partir de su "periodo americano". No solo continuaba yo la genial indicación de Lester en su primera conferencia en Buenos Aires de que la estructura del mundo social es una estructura de grupos, sino también su agudísima observación de que hay en la obra de Schutz un giro que lo distancia de algunas de sus primeras posiciones, y que podríamos asignar - conjeturaba yo- a su "período europeo".

Así fue que, inspirado en Lester, cuestioné que Schutz hubiera sido un individualista metodológico pues concebía la sociología fenomenológica como una ciencia de los grupos, partiendo de la convicción de que el "Nosotros" es primordial con respecto al "yo" y de que la "relación-nosotros" es el fundamento de toda categoría existencial. Remarqué -en obvia cercanía con Lester- que para Schutz la idea de que los individuos pueden existir separados unos de otros es una abstracción ficticia y, por lo tanto, la sociología no se ha de ocupar de individuos sino de membresías grupales. Su objeto serán las relaciones de los individuos en el grupo y de los grupos entre sí, y deberá describir las estructuras internas de cada grupo y sus relaciones con otros; de ahí que tampoco valga decir que los grupos existen aislados unos de otros. 
Seguí refiriéndome a la coerción social en Emile Durkheim, la vigencia en José Ortega y Gasset, las relevancias en Schutz, y la sociología del conocimiento de Karl Mannheim; pero estos son temas que no vienen a colación.

Al finalizar mi ponencia, Lester tomó la palabra para agradecer el reconocimiento y, de algún modo, morigerarlo al decir que yo había ido mucho más allá de lo que él había logrado y que, aunque compartía mi punto de vista, era un desarrollo que me pertenecía. El texto que había leído desmentía el cumplido ya que estaba plagado de citas de Lester. Además, ya le había escuchado elogios de ese tipo en diferentes ocasiones. Más que hablar de mi trabajo, sus palabras hablaban del carácter de Lester, de su cortesía, y de su inquebrantable vocación de alentar a las nuevas generaciones a continuar la reflexión y la práctica de la fenomenología.

No lo sabía entonces, pero esa sería nuestra despedida. La misma vida que me había dado el inesperado privilegio de conocerlo, me regalaba ahora la gracia de poderlo despedir, también de un modo inesperado. Debí haber comprendido que allí se cerraba el círculo de nuestros encuentros y despedidas.

El 8 de mayo de 2016, en el bar de un hotel de Tokio donde incidentalmente coincidimos en el desayuno, aquellos mismos ojos que otrora me miraban desde el sillón de la Academia Nacional de Ciencias de Buenos Aires me contemplaban del mismo modo, sólo que un poco más cansados. Precisamente allí, en los antípodas de mi Buenos Aires, venía a ocurrir esta sigilosa despedida.

Me aconsejó como un padre. Comenzó diciendo, cual si pudiera anticipar su destino accidental y absurdo: I won't be arround here for long. Siguió con una descripción general de cómo son "los fenomenólogos" que, sin embargo, sentía como si fuera una semblanza mía. Tan poco original era mi vida, o tanto me conocía Lester, que me costaba distinguir el plano general del personal en esta caracterización que me ofrecía. Guardo para mí el contenido de sus recomendaciones y de las promesas hechas, ya no tan simples de cumplir. Le debo más que un almuerzo y me lo debo a mí mismo. Estar a la altura de sus consejos es algo que a nadie puede beneficiar en mayor medida que a mí.

Con cada reiterada ausencia suya, en cada encuentro en que nos falta, su figura se agiganta como las sombras al caer la tarde. Lo que vendrá, vendrá con la sombra crepuscular de Lester, en esta aurora boreal en que permanecemos quienes hemos sabido vivir en su luz. 